\title{
Evaluation and reduction of the analytical uncertainties in GC-MS analysis using a boundary regression model
}

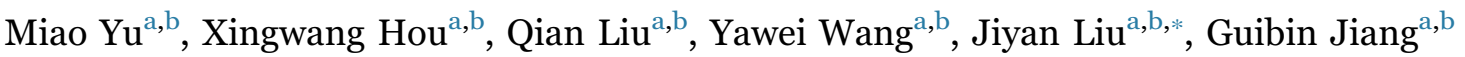 \\ a State Key Laboratory of Environmental Chemistry and Ecotoxicology, Research Center for Eco-Environmental Sciences, Chinese Academy of Sciences, P.O. \\ Box 2871, Beijing 100085, China \\ b College of Resources and Environment, University of Chinese Academy of Sciences, Beijing 100049, China
}

\section{A R T I C L E I N F O}

Keywords:

Data visualization

GC-MS

Environmental analysis

Regression model

\begin{abstract}
A B S T R A C T
The uncertainties in analysis of trace environmental pollutants may come from sample matrix and sample pretreatment process. In this study, a boundary model was developed based on the visualized data of mass spectrometry to evaluate the influences from sample matrix and pretreatment process. The factors affecting the pretreatment procedures, such as the solvents, the extraction sorbents and the extraction process had limited influences compared with matrix effects. Using such boundary model, we found that selecting suitable qualitative and quantitative ions for MS detector is more important for reducing the matrix effect in GC-MS analysis than the traditionally used methods of optimizing the pretreatment process since some clean up sorbents might be useless to reduce the matrix effects. As for 2,2',4,4',5-pentabromodiphenyl ether (BDE-99), the fragmental ions were usually used for qualitative and quantitative analysis, which however was easily affected by the matrix effects. While, molecular ions would eliminate the influences from the sample matrix. Such a model could be used to decrease the uncertainty and increase the accuracy of environmental trace analysis.
\end{abstract}

\section{Introduction}

Various matrices, including water, soil, sediment and biota samples, were analyzed to understand the fate of certain pollutants in the environment [1]. Influences from those matrices affect the sensitivity, selectivity, accuracy and precision in the trace level analysis of pollutants. As one of the most popular environmental analysis method for volatile organic pollutions, gas chromatography-mass spectrometry (GC-MS) is always affected by such influences during sample analysis $[2,3]$. Those matrix effects in GC-MS came from either the co-eluted compounds or the interaction among matrix components, analytes and the instruments $[2-5]$.

Many studies have developed or modified analytical methods, especially the pretreatment methods, to avoid the influences during GC-MS analysis. Some methods, such as chromatography, internal standards, pulsed splitless injection and derivatization, have been involved to improve the quality of the determination $[6,7]$. However, the problems haven't been completely solved and complex pretreatment might not be useful for analysis. For instance, color components can be efficiently removed by using the graphitized carbon black in the clean-up procedure, while this protocol cannot eliminate the matrix enhancement for pesticides in food [2,4].

Limited evaluation method restricted the exploration the nature of the matrix effects. Most of the studies used recovery or certain algorithms to report the matrix effects [8, 9]. However, those methods could not indicate the origins of the influences, and thus could not direct an effective way to eliminate the influences. Therefore, methods were needed to evaluate the sources and the intensities of those influences intuitively.

Data visualization could reveal certain patterns of mass spectral data. For example, the Kendrick mass defect spectrum can be obtained from a high-resolution mass spectrum to show the mass pattern of certain samples [10]. Some excellent softwares such as mzMine and XCMS also supplied certain functions to visualize the mass spectrum data [11,12]. However, to our knowledge, no study has employed such methods to study the matrix effects during the environmental GC-MS analysis.

In this study, visualization methods and mass influence boundary model based on the visualization methods were developed to explore the mass profile during GC-MS full-scan analysis under electron ionization (EI) source. The influences from extraction solvent, pretreatment processes and sample matrixes were also intuitively evalu-

\footnotetext{
* Corresponding author at: State Key Laboratory of Environmental Chemistry and Ecotoxicology, Research Center for Eco-Environmental Sciences, Chinese Academy of Sciences, P.O. Box 2871, Beijing 100085, China

E-mail address: liujy@rcees.ac.cn (J. Liu).
} 
ated. One of the polybrominated diphenyl ethers (PBDEs), which were banned as persistent organic pollutants (POPs) and drew lots of research interests [13], was analyzed as a representative POPs to validate the methods. We hope to find the common properties of matrix effects by data mining and show the implications for environmental analysis.

\section{Material and methods}

\subsection{Materials}

Methylene chloride (DCM) and hexane (chromatographic grade) were obtained from J.T. Baker (Phillipsburg, NJ, USA). Iso-octane (chromatographic grade) was obtained from J \& K Chemical (Newark, DE, USA). Silica gel (100-200 mesh) was acquired from Merck (Darmstadt, Germany). Florisil (60-100 mesh) was purchased from Sigma-Aldrich (St. Louis, MO, USA). Concentrated sulfuric acid (98\%, AR) and neutral alumina (100-200 mesh) were procured from Sinopharm Chemical Reagent (Shanghai, China). Diatomaceous earth was obtained from Restek (Bellefonte, PA, USA).

Silica gel, neutral alumina and florisil were all rinsed with DCM twice firstly. Then silica gel was activated at $180{ }^{\circ} \mathrm{C}$ for $1 \mathrm{~h}$. The $44 \%$ acid silica was prepared by mixing $100 \mathrm{~g}$ of active silica gel with $79 \mathrm{~g}$ of concentrated sulfuric acid. The neutral alumina was baked at $130{ }^{\circ} \mathrm{C}$ for $12 \mathrm{~h}$ and the florisil was held at $130^{\circ} \mathrm{C}$ for 3 days before use. Such steps were used to minimize self-influence. Diatomaceous earth, used as hydromatrix, was rinsed by accelerated solvent extraction (ASE) before use.

Standard reference materials included marine sediment (SRM 1941b) and house dust (SRM 2585) which were obtained from NIST (Gaithersburg, MD, USA), human hair [GBW 07601(GSH-1)], tea leaves (GBW (E) 08001), Tibet soil (GBW 08302) and mya arenaria (GBW 08751) which were obtained from RCEES (Beijing, China). 2,2',4,4',5-Pentabromodiphenyl ether (BDE-99, $50 \mu \mathrm{g} / \mathrm{mL},>99 \%$ ) and 2,4,4',6-Tetrabromodiphenyl ether (BDE-75, $50 \mu \mathrm{g} / \mathrm{mL},>99 \%$ ) was purchased from AccuStandard (New Haven, CT, USA) and gradually diluted to $1 \mu \mathrm{g} / \mathrm{mL}$ in iso-octane before use. ${ }^{13} \mathrm{C}-\mathrm{BDE}-99$ $(50 \mu \mathrm{g} / \mathrm{mL},>99 \%)$ were purchased from Wellington (Guelph, ON, Canada) and gradually diluted to $1 \mu \mathrm{g} / \mathrm{mL}$ in iso-octane before use.

\subsection{Instrument}

An Agilent 6890 GC coupled with a 5973 C MS detector (Agilent Technologies, Palo Alto, CA) and a DB-5 ms column $(15 \mathrm{~m} \times 0.25 \mathrm{~mm}$ $\times 0.1 \mu \mathrm{m}, \mathrm{J} \& \mathrm{~W}$ Scientific, Folsom, CA) was used for analysis. A volume of $2 \mu \mathrm{L}$ of $1 \mu \mathrm{g} / \mathrm{mL}$ standards or $2 \mu \mathrm{L}$ of the samples was injected into the $\mathrm{GC}$ inlet (7683B Series Injector) at $300{ }^{\circ} \mathrm{C}$ in splitless mode. The carrier gas, helium, was controlled at $1.0 \mathrm{~mL} / \mathrm{min}$ (constant flow). The oven program was started at an initial temperature of $100{ }^{\circ} \mathrm{C}$ for $3 \mathrm{~min}$ and then increased to $320^{\circ} \mathrm{C}$ at $10^{\circ} \mathrm{C} / \mathrm{min}$, so the temperature range of interest was from 100 to $320^{\circ} \mathrm{C}$, which is common in environmental analysis. The post run was set at $320^{\circ} \mathrm{C}$ and held for $3 \mathrm{~min}$. Both full scan $(\mathrm{m} / \mathrm{z} 100-1000)$ and SIM modes were performed under EI mode in the mass spectrometer. The solvent delay was 3 min. For each sample, five replicated injections were performed to evaluate the accuracy of determination. The raw data were output as CDF files for further data analysis.

\subsection{Experimental design}

Five groups of samples were analyzed in this study to evaluate the influences from certain sources such as solvents, sorbents, one-step extraction/clean-up pretreatment and real samples. The injections of the air were treated as the baseline data of the whole analysis and used to develop the data visualization method and the boundary model.

Group I. $2 \mu \mathrm{L}$ of the pure DCM, hexane and iso-octane were

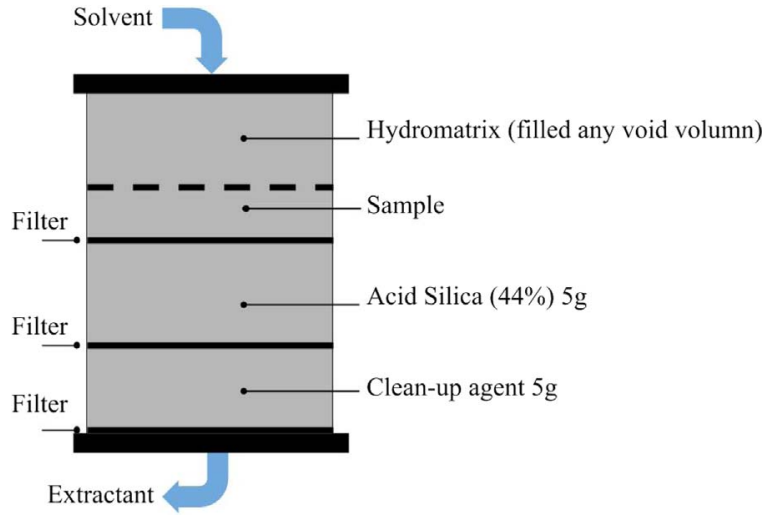

Fig. 1. Packing of the ASE extraction cell for one-step extraction/clean up.

injected into the GC-MS, respectively, to evaluate the influences from the solvents.

Group II. Glass purification columns were filled with $10 \mathrm{~g}$ of neutral alumina, active silica, florisil and diatomaceous earth, respectively, and rinsed with $50 \mathrm{~mL}$ of $1: 1 \mathrm{DCM} /$ hexane. Then another $20 \mathrm{~mL}$ of $1: 1$ $\mathrm{DCM} /$ hexane eluate was collected, evaporated, and re-dissolved into $1 \mathrm{~mL}$ of iso-octane for analysis. This group was used to evaluate the influences origin from the purification sorbents.

Group III. One-step extraction/clean-up pretreatment method modified from published work was employed [14,15]. ASE was performed with $\mathrm{DCM} /$ hexane $(1: 1, v / v)$ at $90^{\circ} \mathrm{C}$ and $1500 \mathrm{psi}$ with 5 -min static time, three static cycles, $60 \%$ flush and a 90 -s purge. The filling of the ASE cell was shown in Fig. 1, but without environmental samples. Five grams of acid silica gel was used as a strong clean-up agent. Five-gram of other polar sorbents, including neutral alumina, active silica, florisil and diatomaceous earth (as blank control), were tested, respectively. The ASE extracts were collected and then concentrated into $1 \mathrm{~mL}$ of iso-octane for injection. This group was used to study the influences of the sorbents during the one-step extraction/ clean-up pretreatment process.

Group IV. Six environmental reference materials, including Tibet soils $(0.5 \mathrm{~g})$, marine sediment $(0.2 \mathrm{~g})$, house dust $(0.2 \mathrm{~g})$, tea leaves $(0.5 \mathrm{~g})$, mya arenaria $(0.5 \mathrm{~g})$ and human hair $(0.5 \mathrm{~g})$, were analyzed by one-step extraction pretreatment to evaluate the influences of the sample matrixes. Here only acid silica was filled together with the sample in the cells and the sorbents were all diatomaceous earth.

Group V. One-step extraction/clean-up pretreatment was used to extract house dust with different purification sorbents (Fig. 1), including neutral alumina, active silica and florisil, to evaluate the effects of different sorbents on the determination.

Finally, BDE-99 was detected as representative pollutant and the method to avoid the matrix influences was explored depend on the boundary model. The amount of $0.2 \mathrm{~g}$ house dust sample (three replicate) was determined for BDE-99 under selected ion monitoring (SIM) mode. Amounts of $20 \mathrm{ng}$ BDE-75 and $25 \mathrm{ng}{ }^{13} \mathrm{C}-\mathrm{BDE}-99$ were used as injection internal standard and surrogate standard, respectively. Limit of detection (LOD) was determined to evaluate the sensitivity of the method. The precision and the accuracy were accessed by the relative standard deviation (RSD) of multiple detections and comparing the quantitative results with the certified value of SRM 2585 , respectively.

\subsection{Data analysis}

Data analysis was performed using R 3.2.3 [16]. Functions were developed for GC-MS data visualization and modeling in which the input function was referenced from the XCMS package $[11,17,18]$. As the results, two kinds of plots and one regression model were obtained to evaluate the responses of the mass spectrums. 


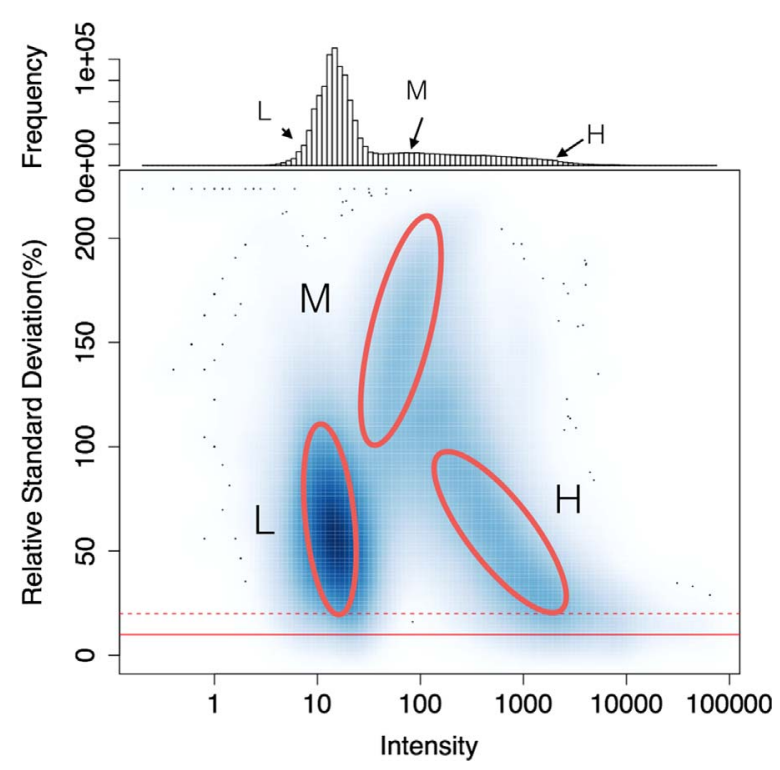

Fig. 2. Smooth scatter plot of mean responses (on a base 10 log scale) versus relative standard deviation (RSD, \%) of five replicated injections of lab air. The histogram on the top is the distribution of the mean responses. The red dashed horizontal line represents RSD at $20 \%$, and the red horizontal line represents the RSD at $10 \%$.

\subsection{Mass spectrum data visualization}

The smooth scatter and the histogram (SSH plots) were used to show the stability of multiple injections. The RSD, axis of ordinates of the smooth scatter, were calculated for mass responses recorded at each time points of replicated GC-MS full-scan of the same sample. The responses with RSDs lower than $20 \%$ and $10 \%$ which includes signals of target and influence compounds should be concerned for the qualitative and quantitative analyses, respectively.

The air samples were injected into the GC-MS and their mass spectrum responses were input into the proposed functions to obtain the visualized data. As shown in Fig. 2, three zones which were named as $\mathrm{L}, \mathrm{M}$ and $\mathrm{H}$ can be found in SSH plot. The $\mathrm{L}$ zone showed a lognormal distribution with high RSDs and low mean responses. Such a distribution is commonly treated as noise signals. The $\mathrm{M}$ zone showed high RSDs and medium mean responses. With the responses increasing, the RSD increased in this zone. The $\mathrm{H}$ zone showed that with increasing responses, the RSD decreased. For the air samples, most of the responses showed RSDs above $20 \%$ and useless for the qualitative and quantitative analysis.

The mass heatmap ( $\mathrm{MH}$ plot) was used to observe the mass intensity distribution along the mass range and retention time of replicated full scans mass data. Also the total ion chromatogram (TIC) were overlapped. Fig. 3 shows a MH plot of the lab air sample data. An inherited influence on the lower mass could be seen. The increase of the baseline in the total ion current was mainly from the responses of relatively high mass. Such a visualization can intuitively show the mass pattern and help to find the mass ranges being easily influenced during a separation process.

\subsection{Mass influences boundary model}

A regression model was developed to formally describe the boundary of the mass affected by various influences. From the SSH plot, responses occurred in zone $\mathrm{L}$ were considered as the noises. The mass threshold can be determined as 100 under which the mass can be given a value of 0 . When the responses were above the threshold, they were given a value of 1 . Then, the given values of the adjacent mass responses were calculated as the finite differences from high to low masses. The lowest mass corresponding to the difference of 1 was

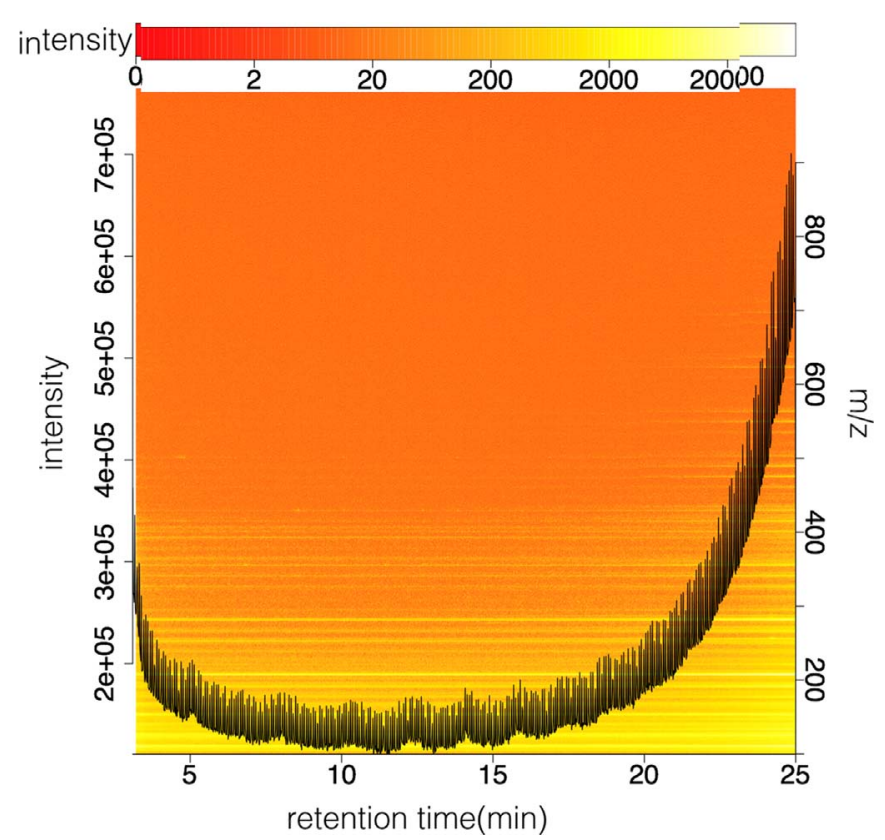

Fig. 3. The total ion chromatogram (TIC) and the heatmap of the mass profile of the mean responses of five injections of lab air. The left axis is the intensity of the total ion chromatogram (TIC), the right axis is the mass. The color relates to the intensity of the mass responses.

recorded as the boundary mass. All of those boundary masses along the retention time were used to make a scatter plot. Because the temperature of a separation program corresponded to the retention time on GC, the retention time could also be transformed into temperature to illustrate the plot.

Ordinary least squares (OLS) and locally weighted scatterplot smoothing (LOWESS) regressions were used to analyze the relationship between the temperature and the boundary mass $[19,20]$. The OLS model described the linear regression but not the non-linear relationship. While the LOWESS model can show a robust or nonlinear relationship between variables that may not be shown in a dominant formula. When those two regression lines showed the same trends, the relationship between temperature and mass was considered as the dominant formula of linear relationship. Generally, larger slope than the baseline data meant that heavier mass was influenced. The intercept showed the mass boundary at the beginning of the data collection. As shown in Fig. 4, the OLS linear model displayed that masses below $\mathrm{m} / \mathrm{z} 137$ suffer great influences, and the boundary mass increases $\mathrm{m} / \mathrm{z} 25$ when the temperature increases $100{ }^{\circ} \mathrm{C}$ on the tested column.

In summary, the SSH plot could be used to find the threshold between noise and signal and the $\mathrm{MH}$ plot show the profile of mass data. The boundary model was built based on the information from SSH and MH plots. The visualization method and the model could be used to evaluate various influences occurred during the GC-MS analysis.

\section{Results and discussion}

\subsection{Influences from the solvents}

The data visualizations of different solvents (group I) were displayed in Fig. S1. Similar to the figures of air sample, SSH plot showed three zones with RSD higher than 20\%. The MH plot also showed high influences at low mass as Fig. 3. The regression model showed that the minor increased boundary had a sharply increase after program temperature increase to $280{ }^{\circ} \mathrm{C}$ (see Fig. S2). However, the intercepts and slopes of the linear regression models were also similar to the air 


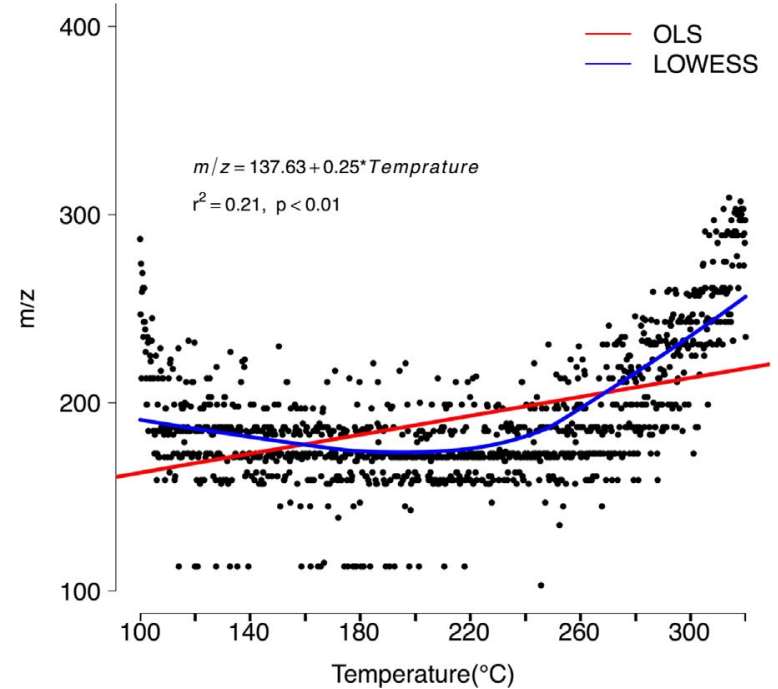

Fig. 4. The boundary model for the influences along the temperature programs. The red line represents the ordinary least squares (OLS) regression line, and the blue line represents the locally weighted scatterplot smoothing (LOWESS) regression line. On the top left is the formula for the OLS regression model, the model's r-squared value and the p-value for the F test.

samples, indicating that little interferences came from tested solvents.

\subsection{Influences from the sorbents}

Influences from the sorbents (group II) were generally considered unavoidable when clean-up process was employed [21-23]. Fig. S3 shows the influences of four commonly used sorbents. The $\mathrm{H}$ zones of different sorbents' SSH plots had more responses than that of solvents. However, such influences were limited, and did not change the mass patterns in the MH plots. Sorbents increased the intercepts and slopes of the boundary regression lines (see Fig. S4) compare with solvents. For active silica gel, the slope was 0.7 mass per degree Celsius, significantly higher than other sorbents which showed similar slopes with the solvents. Most of the compounds recorded in the NIST 14 mass spectrum database show total mass range from m/z 100 to 500 [24]. When detecting the mass of $\mathrm{m} / \mathrm{z} 300$ of a target compound with retention time around $20 \mathrm{~min}$, it would suffer influences from the silica gel pretreatment. Other polar sorbents might be safe from those influences and should be preferred to remove polar inferences rather than silica gel when they showed similar clean up capability for certain analyte. In a word, the influences from the sorbents with a exception of silica gel were also limited and at the same level with the influences from solvents.

\subsection{Influences from the one-step extraction/clean-up pretreatment process}

One-step clean/extraction by ASE with the sorbents filled into extraction cells was commonly used in environmental analysis [14,25]. As shown in Figs. S5 and S6, more responses occurred in zone $\mathrm{H}$ of samples in group III than that of group II. The boundary models also seemed similar to the test for sorbents. The pressure in the ASE cells would improve the extraction efficiency and also might bear responsibility for the influences on lower mass. To protect the instrument from more pollution, such pretreatment was necessary. And the one-step clean/extraction with the sorbents filled into extraction cells also showed similar influences compared with group II, which is limited.

\subsection{Influences from the sample matrix}

Six different environmental matrices (group IV) were employed to explore the matrix influences. As shown in Fig. 5, SSH plots showed much more responses in zone $\mathrm{H}$. Some RSD of the responses in zone $\mathrm{H}$ were lower than $10 \%$, which would affect the qualitative analysis. The TICs of different sample matrixes were different and the raise of baseline was not so clear at a higher temperature. However, the $\mathrm{MH}$ plot and the boundary models showed similar mass patterns (see Fig. 6). The slopes of the linear regression were much larger than those of former groups while the intercepts were relatively small. Tibet soil was relatively clean while the other matrices made the mass boundary increase shapely along the temperature program. Such patterns showed greater influences than other groups. Thus the matrix effect from the samples might be the main interference sources of environmental analysis.

Using the house dust, we also evaluated different additional sorbents during the one-step clean/extraction ASE method (group V). The results (see Fig. S7, Fig. S8) showed no improvement for elimination the matrices influence on the analysis of SRM 2585. Some sorbents might remove the impurities, but the impurities may have no signals on GC-MS at all. Also more steps may increase the uncertainty of the analysis procedure. The data visualization and boundary model would be helpful for environmental analysis to reduce unnecessary clean-up steps.

It showed that the matrix effect could be described by the mass boundary model. If the qualitative and quantitative ion masses of target compound were under the boundary, the analysis would suffer severe influences. Therefore, monitoring the ions with mass higher than mass boundary or making the analytes elute at lower temperature to decrease the boundary mass would help to avoid the interferences. Traditionally, employing a short column or using derivatization are effective ways to change the elution temperature of the analytes on GC.

\subsection{Elimination of the matrix effects}

Using pure standards to establish the instrument method is common in environmental analysis. However, it did not consider the potential influences from the matrix effects. If developing a mass boundary model firstly, it is easy to evaluate and control the matrix effects during the environmental analysis.

BDE-99 was one of the major congeners of PBDEs commonly found in environmental samples $[13,26]$. In SRM 2585, BDE-99 appears at a high concentration with the reference value of $892 \pm 53 \mathrm{ng} / \mathrm{g}$. Previous methods for BDE-99 analysis generally much preferred to use fragmental ions with loss of two bromine atoms $(\mathrm{m} / \mathrm{z}(404,406)$ as the qualitative and quantitative ions compared with molecular ions $(\mathrm{m} / \mathrm{z}$ 564, 566) [27], as shown in Fig. 7. However, the influence boundary at retention time of BDE-99 in real sample was approximately m/z 460 according to the linear regression in Fig. $6 \mathrm{C}$, which is lower than the molecular ions and higher than the fragmental ions of BDE-99. Selecting the fragmental ions as the qualitative and quantitative ions would make the quantitative trace analysis inaccurate. However, selecting the molecular ions for analysis which was larger than the boundary would avoid the influences from the matrix and decrease uncertainties from real samples. In addition, choosing the ions with higher $\mathrm{m} / \mathrm{z}$ values are always recommended to decrease the matrix effects.

To evaluate the contribution of the boundary model to decrease the uncertainties of the detection, LODs obtained using fragmental ions and molecular ions were determined and compared. LODs determined based on fragmental ion was $31 \mathrm{ng}$ and those based on molecular ion was $12 \mathrm{ng}$. Selecting molecular ions according to the boundary model significantly increase the sensitivity of the detection. As for the quantification of BDE-99 in SRM $2585(892 \pm 53 \mathrm{ng} / \mathrm{g})$, the concentration detected by the molecular ion was $896 \pm 9 \mathrm{ng} / \mathrm{g}$ and detected by fragmental ion was $940 \pm 8 \mathrm{ng} / \mathrm{g}$. A little bit better accuracy was obtained using molecular ion for quantification. The RSD of the results from molecular ions was $0.97 \%$, while from the fragmental ion was 

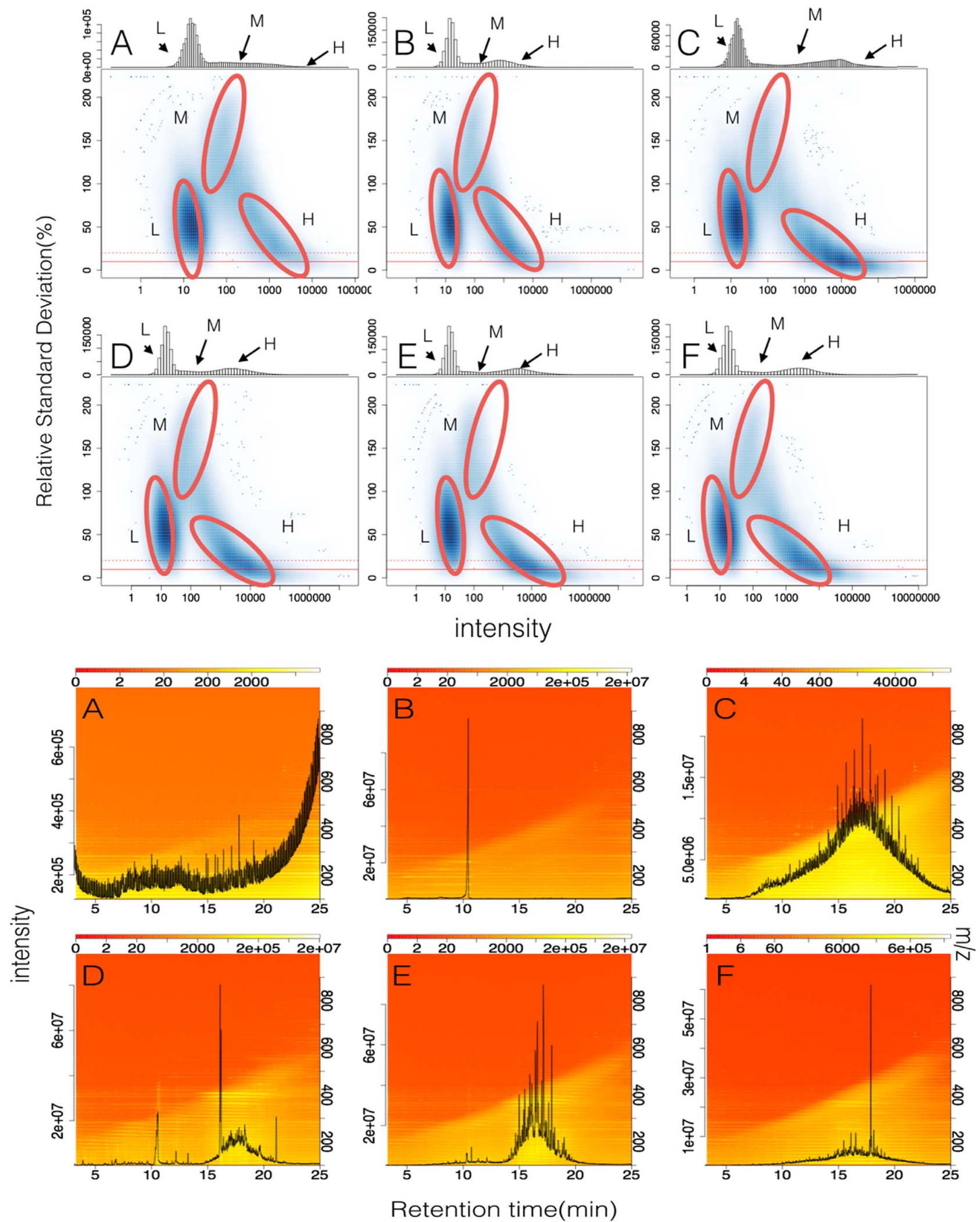

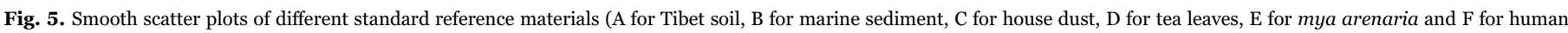
hair).

$0.86 \%$. The precisions obtained from molecular and fragmental ions were merely the same. In summary, using molecular ions as the qualitative and quantitative ions, the sensitivity and the accuracy were better than using fragmental ions. The boundary model directed to decrease the uncertainties origin from matrix effects and get better results in trace analysis.

\section{Conclusion}

In this study, we proposed data visualization methods for GC-MS 


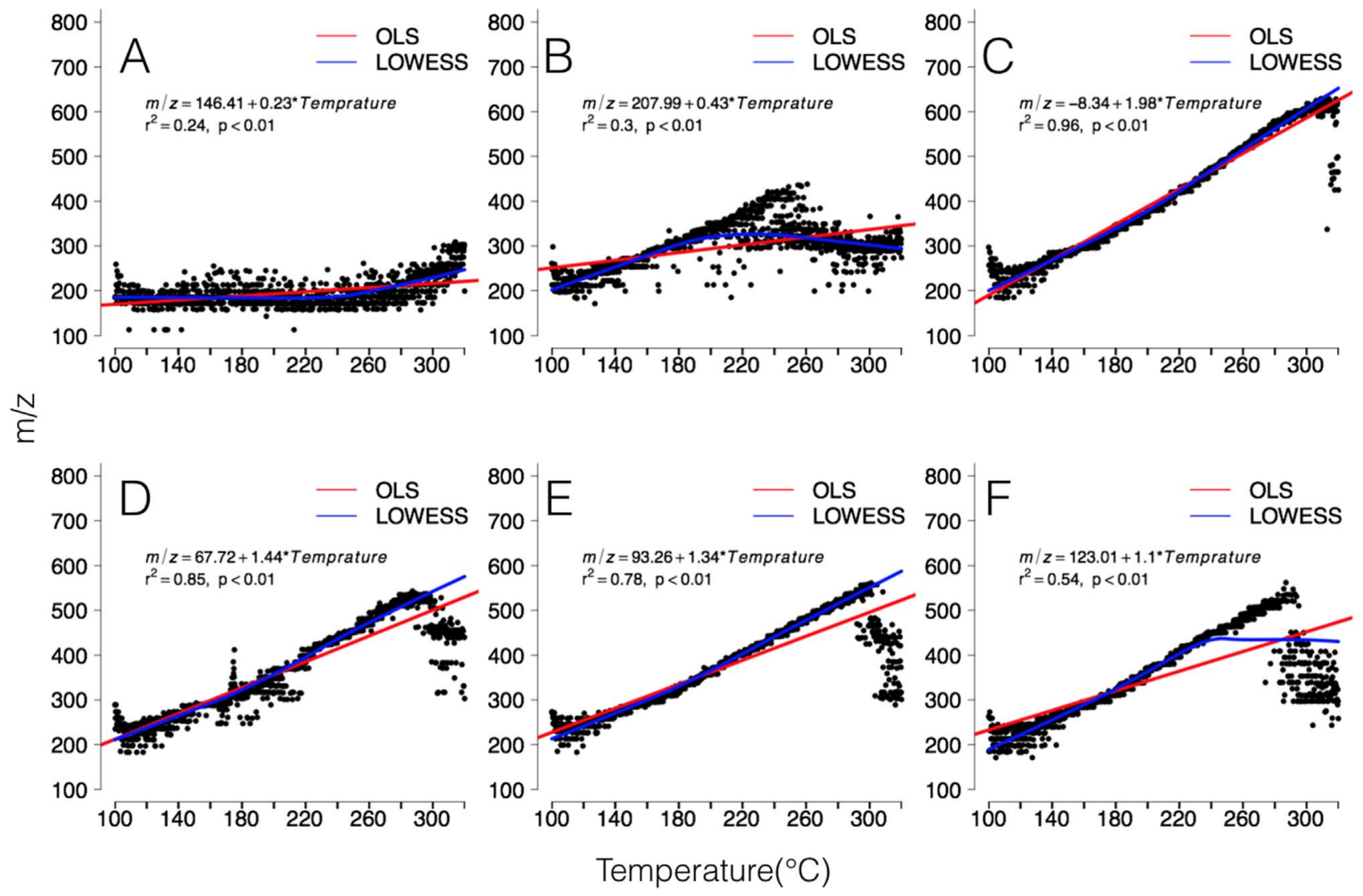

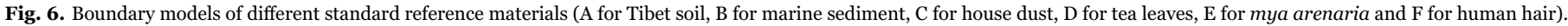

\section{BDE-99}

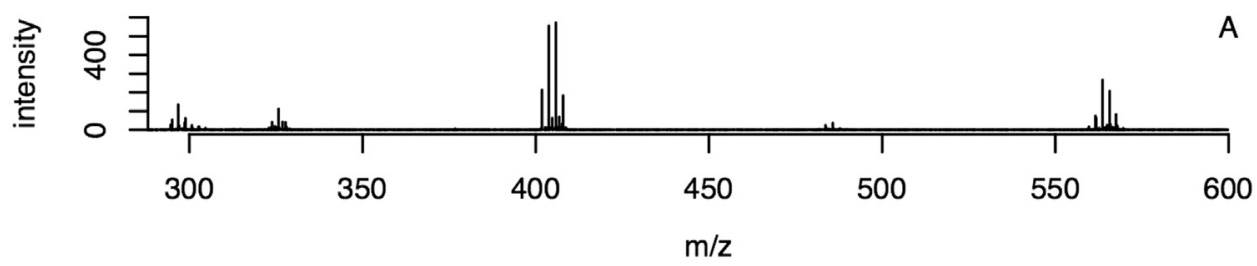

$\mathrm{m} / \mathrm{z}=564$

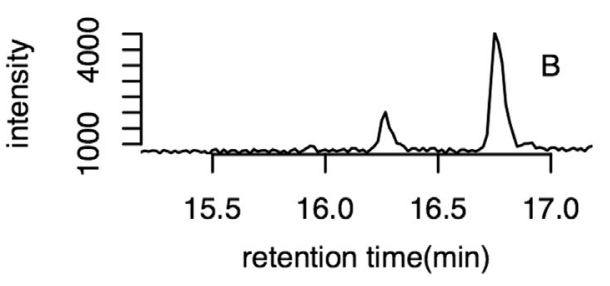

$\mathrm{m} / \mathrm{z}=404$

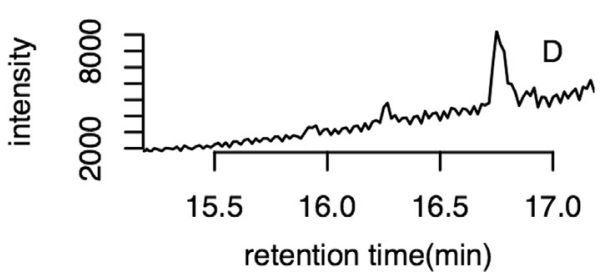

$\mathrm{m} / \mathrm{z}=566$

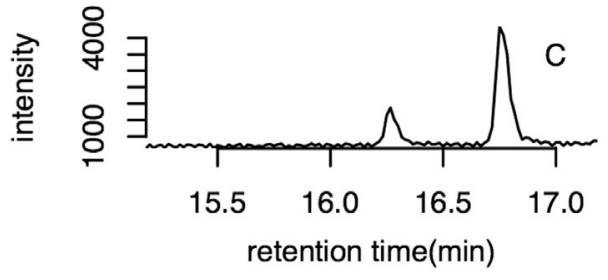

$\mathrm{m} / \mathrm{z}=406$

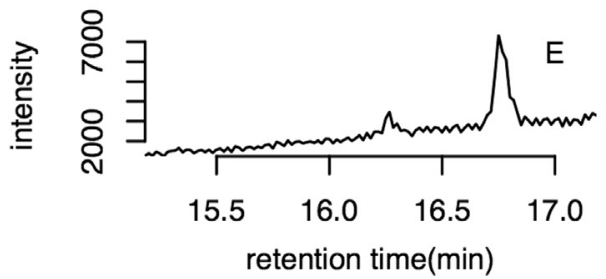

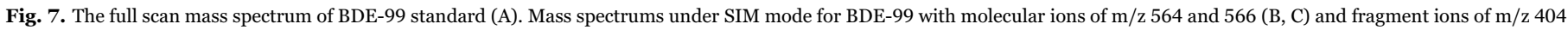
and 406 (D, E) in house dust. The retention time of BDE-99 was $16.8 \mathrm{~min}$. 
data and a boundary model to evaluate the influences during environmental analysis. Those methods could help us find solutions for the matrix effects, selection of the qualitative and quantitative ions, and reduce the pretreatment steps. This line of thinking is totally different from the traditional pretreatment optimization methods.

\section{Supporting Information}

This material is available free of charge via the Internet.

\section{Acknowledgment}

This work was supported by the National Key Basic Research Program of China (2014CB441105), the National Natural Science Foundation of China (21277153), and Chinese Academy of Science (XDB14010400).

\section{Appendix A. Supporting information}

Supplementary data associated with this article can be found in the online version at doi:10.1016/j.talanta.2016.11.046.

\section{References}

[1] L.H. Keith, W. Crummett, J. Deegan, R.A. Libby, J.K. Taylor, G. Wentler, Anal. Chem. 55 (1983) 2210-2218.

[2] J. Hajslova, K. Holadova, V. Kocourek, J. Poustka, M. Godula, P. Cuhra, M. Kempny, J. Chromatogr., A 800 (1998) 283-295.

[3] F.J. Santos, M.T. Galceran, J. Chromatogr. A 1000 (2003) 125-151.
[4] D.R. Erney, T.M. Pawlowski, C.F. Poole, J. High. Resolut. Chromatogr. 20 (1997) $375-378$.

[5] J. de Boer, R.J. Law, J. Chromatogr., A 1000 (2003) 223-251.

[6] D.R. Erney, C.F. Poole, Hrc-J. High. Res Chromotogr. 16 (1993) 501-503.

[7] F.J. Schenck, S.J. Lehotay, J. Chromatogr., A 868 (2000) 51-61.

[8] M.V. Salvia, C. Cren-Olive, E. Vulliet, J. Chromatogr., A 1315 (2013) 53-60.

[9] C. Rimayi, D. Odusanya, F. Mtunzi, S. Tsoka, Chemosphere 118 (2015) 35-43.

[10] P.G.A. Pedrioli, J.K. Eng, R. Hubley, M. Vogelzang, E.W. Deutsch, B. Raught, B. Pratt, E. Nilsson, R.H. Angeletti, R. Apweiler, K. Cheung, C.E. Costello, H. Hermjakob, S. Huang, R.K. Julian, E. Kapp, M.E. McComb, S.G. Oliver, G. Omenn, N.W. Paton, R. Simpson, R. Smith, C.F. Taylor, W.M. Zhu, R. Aebersold, Nat. Biotechnol. 22 (2004) 1459-1466.

[11] C.A. Smith, E.J. Want, G. O'Maille, R. Abagyan, G. Siuzdak, Anal. Chem. 78 (2006) 779-787.

[12] T. Pluskal, S. Castillo, A. Villar-Briones, M. Oresic, Bmc Bioinform. 11 (2010).

[13] Governments unite to step-up reduction on global DDT reliance and add nine new chemicals under international treaty, 〈http://chm.pops.int/Convention/Media/ PressReleases/COP4Geneva,9May 2009/tabid/542/Default.aspx>, (accessed Jul 7, 2016).

[14] M.A. Abdallah, D. Drage, S. Harrad, Environ. Sci.-Process. Impacts 15 (2013) 2279-2287.

[15] E. Bjorklund, A. Muller, C. von Holst, Anal. Chem. 73 (2001) 4050-4053.

[16] R.C. Team, in, 2015, 〈https://www.R-project.org/〉.

[17] H.P. Benton, E.J. Want, T.M.D. Ebbels, Bioinformatics 26 (2010) 2488-2489.

[18] R. Tautenhahn, C. Bottcher, S. Neumann, Bmc Bioinform. 9 (2008).

[19] W.S. Cleveland, J. Am. Stat. Assoc. 74 (1979) 829-836.

[20] W.S. Cleveland, Am. Stat. 35 (1981) (54-54).

[21] A. Beyer, M. Biziuk, Food Res. Int. 43 (2010) 831-837.

[22] K. Grob, J. Chromatogr. 287 (1984) 1-14.

[23] T. Hyotylainen, Anal. Bioanal. Chem. 394 (2009) 743-758.

[24] Us Department of Commerce, N., NIST Standard Reference Database 1A v14.

[25] E. Cocco, C. Guignard, L. Hoffmann, T. Bohn, Int. J. Environ. Anal. Chem. 91 (2011) 333-347.

[26] C.A. de Wit, Chemosphere 46 (2002) 583-624.

[27] R.A. Hites, Environ. Sci. Technol. 42 (2008) 2243-2252. 